\title{
The role of elastography in the assessment of chronic liver disease in children
}

\author{
Süleyman Sönmez ${ }^{1}$, Merve Boşat ${ }^{2}$, Nihal Yurtseven ${ }^{3}$, Eray Yurtseven ${ }^{4}$
}

1. Department of Radiology, Bakirkoy Dr. Sadi Konuk Training \& Research Hospital.

2. Department of Healthcare Management, Faculty of Health Sciences, Bezmialem Vakıf University.

3. Faculty of Sport Sciences, Istanbul University-Cerrahpaşa, Turkey.

4. Department of Biostatistics, Istanbul Faculty of Medicine, Istanbul University, Turkey.

\begin{abstract}
Background: Conventional ultrasonography is a method preferred for the investigation of chronic liver diseases in pediatric groups, as it is non-invasive, cheap, feasible and available. The purpose of this study is to present the role of Share-wave Elastography (SWE) in terms of diagnostic value in children diagnosed with "chronic liver disease."

Methods: We studied patients who had been diagnosed with chronic liver disease between March 2012-September 2015, and who had undergone liver biopsy and had their pathology results, compared with 26 healthy subjects. Statistical analysis was performed with IBM SPSS Statistics for Windows, Version 20.0. "Pearson Correlation Analysis" was performed in order to measure the relationship between elastography values and Brunt level.

Results: This study had 107 subjects in total, consisting of 81 patients between 0-204 months of age Pearson correlation coefficient level was determined as $r=0.644$. Since the correlation coefficient is positive, there is a same-directional relationship between Elastography level and Brunt degree. This means that while one of the variables is increasing, the other one will also increase.

Conclusion: Since it is known that development of hepatic fibrosis is a dynamic process, and that many hepatic fibrosis etiologies are known to continue throughout the course of life, the application of Real time SWE method instead of repeated liver biopsies on patients is a much simpler and smart method. Increasing the clinical use of Real Time SWE method with future studies might provide an opportunity for preventing unnecessary liver biopsies since the patients are evaluated in a shorter time and in a cost-effective manner.
\end{abstract}

Keywords: Shear-Wave Elastography, Brunt degree, chronic liver disease, liver biopsy.

DOI: https://dx.doi.org/10.4314/ahs.v19i3.57

Cite as: Sönmez S, Boşat M, Yurtseven N, Yurtseven E. The role of elastography in the assessment of chronic liver disease in children. Afri Health Sci. 2019;19(3): 2806-2811. bttps:// dx.doi.org/10.4314/abs.v19i3.57

\section{Background}

Chronic liver disease is a significant health issue among children and it can result in fibrosis, cirrhosis and liver failure. Since the prognosis and monitoring of these diseases are different from each other, distinguishing them is important. ${ }^{1,2}$. While liver needle biopsy is still recognized to be the gold standard for the assessment of liver

\section{Corresponding author:}

Süleyman Sönmez

Bakirkoy Dr. Sadi Konuk Training

\& Research Hospital, İstanbul /Turkey

Tel: $+90(2124147171$

E-mail: suleymansonmez84@gmail.com fibrosis in the diagnosis and follow-up of chronic liver diseases, researchers tend to study non-invasive methods in the determination of liver fibrosis, as liver needle biopsy is an invasive, painful and expensive procedure, which can cause possible sampling errors and potential complications $^{3,4}$. Conventional ultrasonography is preferred for the investigation of chronic liver diseases in children, as it is non-invasive, cheap, feasible and available. Elastography, an ultrasonographic non-invasive procedure that measures tissue stiffness ${ }^{5,6}$. Liver fibrosis is a result of chronic damage, and attacks and remissions throughout the course of the disease trigger the formation of fibrosis. Apart from being a direct indicator of liver damage, fibrosis has an important role in the development of hepatocyte dysfunction and portal hypertension. In a clin-

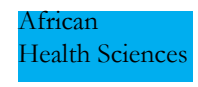

C 2019 Sönmez et al. Licensee African Health Sciences. This is an Open Access article distributed under the terms of the Creative commons Attribution
License (https://creativecommons.org/licenses/BY/4.0), which permits unrestricted use, distribution, and reproduction in any medium, provided the
original work is properly cited. 
ical aspect, knowing the state and progress of fibrosis is important in the assessment of treatment response for the severity and the prognosis of the disease. The degree of fibrosis in the liver is important in the determination of prognosis, and management of the process in chronic liver disease. Patients with fibrosis benefit from treatment in case of early diagnosis ${ }^{7,8}$. Shear-Wave Elastography is a non-invasive method, and the purpose of this study was to compare Real Time Shear-Wave Elastography method, with histopathological Brunt scoring systems, which are recognized to be the diagnostic reference method, and to determine its suitability for routine use in cases with chronic liver disease in the assessment of liver fibrosis. In summary, the purpose of this study was to present the role of Share-wave Elastography, in terms of diagnostic value in children diagnosed with "Chronic Liver Disease" ${ } 9,10$.

\section{Patients and methods}

We studied 107 subjects in total. These consisted of 81 patients aged between 0-204 months, and who attended Istanbul University Cerrahpaşa Faculty of Medicine Department of Pediatrics, Division of Pediatric Gastroenterology, Hepatology and Nutrition between March 2012-September 2015. They were diagnosed with chronic liver disease, underwent liver biopsy and had their pathology results. We also studied 26 healthy cases.

"Real time Shear-wave Elastography" was performed on patients in the study groups at Istanbul University Cerrahpaşa Faculty of Medicine Department of Radiology within the same period, and all results were evaluated retrospectively. Patients older than 204 months, those who did not undergo liver biopsy and "Real time Shear-wave Elastography" procedure were excluded. The control group was selected from the children who had attended for reasons other than liver disease and Medical Ultrasonography.

All SWE examinations were performed with "Aixplorer ultrasound system Super Sonic Imagine SA, Aix-enProvence, France convex broadband probe SC6-1".
Patients were asked to not eat anything, starting from at least 8 hours before the procedure, due to the fact that hepatobiliary ultra-sonography was applied before Real Time Shear-Wave Elastography procedure and B-mode imaging was used as a guide during Real Time Shear-Wave Elastography. No drugs were administered to patients for imaging throughout the process, and no radiation administration was performed since this method is performed with an ultrasound device. In the procedure, first B-mode imaging was performed for liver imaging, and then it was switched to elastography mode in synchronization with B-mode imaging. During elastography procedure, shearwaves were formed with successive pressures applied to the skin with the ultrasound probe. Statistical analysis was performed with IBM SPSS Statistics for Windows, Version 21.0 IBM Corp., Armonk, NY; licence number: eb3501007d98f50286ae. "Receiver Operating Characteristics Curve ROC"method was used in order to determine the cut- off value. The diagnostic performance of elastography and the distinction between histopathological fibrosis stages were evaluated with respect to the areas under the ROC curve AUROC: areas under the ROC curve, accuracy. Since the data is distributed homogeneously, "Independent Sample - t test" and "One-way ANOVA" analyses were used for the comparison between groups. A p value of $<0.05$ was assumed to be statistically significant. "Pearson Correlation Analysis" was performed in order to measure the relationship between elastography values and Brunt level.

Ethical approval was obtained form Istanbul University Cerrahpaşa Faculty of Medicine Clinical Trials Ethics Committee Number: 83045809/604.01/02-394991.

\section{Results}

From the 107 subjects in total, 81 patients were diagnosed with chronic liver disease and 26 health cases. The minimum age of the patients who participated in the study was 0 months, and the maximum was 204 months.

Brunt classification and elastography measurement results of patients who had undergone liver biopsy and had their pathology results are presented in Table 1. 
Table 1: Distribution of brunt classification and elastography measurement values

\begin{tabular}{|c|c|c|c|c|c|c|}
\hline Brunt Level & $\mathbf{N}$ & Average $\mathrm{kPa}$ & $\begin{array}{l}\text { Standard } \\
\text { Deviation }\end{array}$ & Standard Error & Minimum & Maximum \\
\hline Fo & 17 & 9.90 & 6.14 & 1.49 & 5.80 & 31.90 \\
\hline F1 & 18 & 16.63 & 9.60 & 2.26 & 7.10 & 36.50 \\
\hline F2 & 19 & 18.20 & 10.04 & 2.30 & 6.50 & 42.40 \\
\hline F3 & 12 & 20.26 & 5.78 & 1.67 & 12.10 & 28.90 \\
\hline F4 & 15 & 25.32 & 6.51 & 1.68 & 16.40 & 42.30 \\
\hline
\end{tabular}

Patients who have F0 values in the patient group were compared with the values of control group. Since the data is distributed homogeneously, "Independent sample- t test" was applied to these groups. No significant difference was determined between these groups Sig $\mathrm{p}>0,05$. Patient group was firstly subjected to "One-way
ANOVA" test in order to investigate whether there was a difference between the groups with regard to a single characteristic. Since intergroup variances are distributed homogeneously, calculations were proceeded with "Posthoc Tukey" test. $\mathrm{P}<0,05$ is recognized to be significant and presented in Table 2.

\begin{tabular}{|c|c|c|c|c|c|c|}
\hline \multirow{2}{*}{\multicolumn{2}{|c|}{ Brunt Classification }} & \multirow{3}{*}{\begin{tabular}{|l} 
Mean difference $1-J$ \\
-6.73301
\end{tabular}} & \multirow{3}{*}{\begin{tabular}{|l} 
Stc. error \\
2.72967
\end{tabular}} & \multirow{3}{*}{\begin{tabular}{|l} 
Sig. \\
109
\end{tabular}} & \multicolumn{2}{|c|}{$95 \%$ confidence Interval } \\
\hline & & & & & \multirow{2}{*}{\begin{tabular}{|l} 
Lower bound \\
$-14,3607$ \\
\end{tabular}} & \multirow{2}{*}{\begin{tabular}{|l} 
Upper bound \\
.8946
\end{tabular}} \\
\hline \multirow{4}{*}{ Fo } & F1 & & & & & \\
\hline & F2 & $-8.29938^{\circ}$ & 2.69455 & .023 & \begin{tabular}{|l|l}
-15.8289 \\
\end{tabular} & .7699 \\
\hline & F3 & $-10.36078^{\circ}$ & 3.04313 & .009 & \begin{tabular}{|l|}
-18.8664 \\
\end{tabular} & -1.8572 \\
\hline & F4 & \begin{tabular}{|l|l|}
$-15.441412^{\circ}$ \\
\end{tabular} & 2.85918 & .000 & \begin{tabular}{|c|c|}
-23.4037 \\
\end{tabular} & -7.4246 \\
\hline \multirow{3}{*}{$F_{1}$} & Fo & 6.73301 & 2.72967 & .109 & \begin{tabular}{|l}
.8946 \\
\end{tabular} & 14.3607 \\
\hline & F2 & -1.56637 & 2.65476 & .976 & $\begin{array}{l}-8.9847 \\
\end{array}$ & 5.8519 \\
\hline & F3 & -3.62778 & 3.00795 & .748 & -12.0330 & 4.7775 \\
\hline & $\mathrm{F} 4$ & \begin{tabular}{|l}
$-8.6811^{\circ}$ \\
\end{tabular} & 2.82171 & .024 & \begin{tabular}{|l|l|}
-16.5659 \\
\end{tabular} & .7963 \\
\hline \multirow{3}{*}{$\mathrm{F}_{2}$} & Fo & $8.29938^{\circ}$ & 2.69455 & .023 & .7699 & 15.8289 \\
\hline & $F 1$ & 1.56637 & 2.65476 & .976 & -5.8519 & 8.9847 \\
\hline & F3 & -2.06410 & 2.97612 & .958 & -10.3777 & 6.2549 \\
\hline
\end{tabular}




\begin{tabular}{|c|c|c|c|c|c|c|}
\hline & $\mathrm{F} 4$ & -7.1.1474 & 2.78775 & .090 & \begin{tabular}{|l|l|}
-1490047 \\
\end{tabular} & .6752 \\
\hline \multirow{4}{*}{ F3 } & Fo & $10.36078^{\circ}$ & 3.04313 & $\mid .009$ & 1.5572 & 18.8644 \\
\hline & $\mathrm{F} 1$ & 3.62778 & 3.00795 & .748 & -4.7775 & 12.0330 \\
\hline & $\mathrm{F} 2$ & 2.06140 & 2.97612 & .958 & -6.2549 & $\mid$\begin{tabular}{|l|l|}
10.3777 \\
\end{tabular} \\
\hline & F4 & -5.05333 & 3.12595 & .492 & \begin{tabular}{|l|l}
-13.7883 \\
\end{tabular} & 3.6817 \\
\hline \multirow{4}{*}{ F4 } & Fo & |15.441412 & 2.85918 & .000 & \begin{tabular}{|l}
7.42466 \\
\end{tabular} & 23.4037 \\
\hline & $\mathrm{F} 1$ & $8.68111^{\circ}$ & 2.82171 & $\mid .024$ & .7963 & |16.5659 \\
\hline & $\mathrm{F2}$ & \begin{tabular}{|l}
7.111474 \\
\end{tabular} & 2.78775 & .090 & .6752 & \begin{tabular}{|l|l}
14.9047 \\
\end{tabular} \\
\hline & F3 & 5.05333 & 3.12595 & .492 & \begin{tabular}{|l|l|}
-3.6817 \\
\end{tabular} & \begin{tabular}{|l|l}
13.7883 \\
\end{tabular} \\
\hline
\end{tabular}

"Pearson Correlation Analysis" was performed in order to measure the relationship between elastography values and Brunt level. Pearson correlation coefficient level was determined as $r=0.644$. Since the correlation coefficient is positive, there is a same-directional relationship between Elastography level and Brunt degree. This means that while one of the variables is increasing, the other one will also increase.

\section{Discussion}

Liver biopsy is still the the gold standard in the diagnosis and assessment of liver fibrosis ${ }^{11,12}$. This procedure is costly, invasive and carries risk of complications. It needs to be performed in the company of a senior physician ${ }^{13}$. Furthermore, there are limiting factors such as sampling errors, and differences in the assessment among pathologists who examine the biopsy material ${ }^{14,15}$. Ferraioli et al. ${ }^{16}$. established that the cut-off value of Brunt groups of F2 and above should be $7.1 \mathrm{kpa}$ and above, while it should be $8.7 \mathrm{kpa}$ for groups F3 and above, and $10.4 \mathrm{kpa}$ for patients with F4 fibrosis. Since the patient group includes adult Hepatitis C patients in this study, it is considered that the results might be different from these findings in our study, which had children with chronic liver disease ${ }^{16}$. Myers et al. ${ }^{17}$ have reported 56\%-96\% sensitivity and $66 \%-91 \%$ specificity in the determination and scoring of liver fibrosis in their multi-center study of 251 cases performed using Transient Elastography method to assess liver fibrosis in Chronic Hepatitis B, Chronic Hepatitis C and NASH patients. They took liver biopsy and METAVIR scoring as reference. Their results had quantitative value, similar to Real Time SWE method. As a result of their study, they asserted that Transient Elastography method had an important role in excluding cases with stage F3 bridging fibrosis and F4 cirrhosis. However, they stated that it was insufficient for the determination of stage F2 significant fibrosis, similar to the findings of Ferraioli.

Sporea et al. demonstrated a strong linear relationship between fibrosis and Transient Elastography in their study assessing of liver stiffness. Liver biopsy and METAVIR scoring were also taken as reference in this study, and obtained values had quantitative characteristics.

For determining $\mathrm{F} \geq 2$ fibrosis values, we obtaiened better results than those reported by Ferraioli et al. ${ }^{17}$ and Myers et al. ${ }^{18}$. Leschied et al.2015 determined significantly high elastography values in their study performed on patients with biliary atresia. They stated that "pre-operative elastography is an emerging non-invasive application and a method to provide benefit in pediatric group' with biliary atresia ${ }^{19}$. While in our study, a high correlation was found between elastography values and fibrosis level, in operated patients with biliary atresia, Corpechot et al. 2006 found 84\% correlation between liver stiffness value and fibrosis, and $79 \%$ correlation in histological 
classification in their study performed with patients with primary and secondary sclerosing cholangitis. They obtained the same results when PBC and PSC patients were subjected to correlation analysis separately ${ }^{20}$. In our study, liver elasticity of the group with fibrosis that was proven with biopsy result were determined to be significantly different. With these results, $64 \%$ correlation was found between elastography value and fibrosis. No significant difference was found between the control and patient group with F0 result. This suggests that findings of liver in early stage fibrosis and healthy liver are similar with regard to measurement values, and coming to a conclusion with elastography assessment is difficult. Similarly, Hanquinet et al. have reported that elastography values increase with progressing fibrosis level, nevertheless there are some limitations in the assessment of first stages of fibrosis ${ }^{21}$. On the other hand, values of $98 \%$ in congenital hepatic fibrosis, 93\% in aAutoimmune hepatitis, 90\% in operated bile duct atresia, 89\% in chronic hepatitis B, $88 \%$ in depot disease, $77 \%$ in Criptogenic Disease, $67 \%$ in Cystic Fibrosis, 52\% in Wilson's disease, 45\% in Hepatosteatosis were found between elastography values of the sub-groups in our study and fibrosis.

In our study, a high correlation was observed between elastography value and degree of degree fibrosis particularly in inflammatory processes and cholestatic diseases, while this correlation was observed to be lower in Wilson's disease, hepatosteatosis and depot diseases. The reason of the decrease in these diseases is considered to be especially due to hepatosteatosis experienced in early stage. Since there is no extensive study on depot diseases in the literature, elastography studies performed on children with depot disease will be valuable in providing important information. Unlike the single-point measurement in liver biopsy, there are advantages of Real Time SWE method such as performing measurements on numerous points in right and left lobes of liver parenchyma by placing as many ROIs as possible in liver parenchyma, and examining a larger area in the liver.

\section{Conclusion}

Since it is known that develpoment of hepatic fibrosis is a dynamic process, and that many hepatic fibrosis etiologies are known to continue throughout the course of life, the application of Real time SWE method instead of repeated liver biopsies on patients is a much simpler and smart method. Increasing the clinical use of Real Time SWE method with future studies might provide an opportunity for preventing unnecessary liver biopsies since the patients are evaluated in a shorter time and in a cost-effective manner.

\section{Conflict of ineterst}

None declared.

\section{References}

1. Campbell MS, Reddy KR Review article: The evolving role of liver biopsy. Aliment Pharmacol Ther. 2004;20:249259 PubMed Doi:10.1111/j.1365-2036.2004.02071.x

2. Garcia-Tsao G, Boyer JL. Out-patient Liver Biopsy: How safe is it? Ann Intern Med. 1993;118:150-153 PubMed DOI: 10.7326/0003-4819-118-2-199301150-00013

3. Rockey DC, Bissell DM. Non-invasive Measures of Liver Fibrosis. Hepatology. 2006; 43: 113-120 PubMed DOI 10.1002/hep.21046

4. Garcia-Tsao G, Boyer JL. Out-patient Liver Biopsy: How safe is it? Ann Intern Med. 1993;118:150-153 PubMed DOI: 10.7326/0003-4819-118-2-199301150-00013

5. Wells PN, Liang HD 2011 Medical ultrasound: imaging of soft tissue strain and elasticity. J R Soc Interface. 8:15211549 DOI: 10.1098/rsif.2011.0054

6. Rivaz H, Boctor EM, Choti MA, Hager GD. 2014. Ultrasound elastography using multiple images. Medical image analysis, 182, 314-329. https://doi.org/10.1016/j. media.2013.11.002

7. Afdhal NH, Nunes D. Evaluation of liver fibrosis: a concise review. Am J Gastroenterol 2004;99:1160-74 PubMed Afdhal NH, Nunes D. Evaluation of liver fibrosis: a concise review. Am J Gastroenterol. 2004;99:116074 PubMed.

8. Friedman SL. Liver fibrosis-from bench to bedside. J Hepatol. 2003;38:38-53 PubMed .https://doi. org/10.1016/S0168-82780200429-4

9. Baron R L, Freeny PC, Moss A A, The liver. In: Moss AA Ed., Computed Tomography of the Body with Magnetic Resonans Imaging. ${ }^{\text {nd }}$. ed. Philadelphia. W.B.Saunders; 1992:735-760

10. Carol M Rumack, M.D, SR Wilson MD, JW Charboneau MD. Diagnostic Ultrasound. Vol.1:59-61

11. Bravo AA, Sheth SG, Chopra S: Liver biopsy. $N$ Engl J Med. 2001, 344: 495-500 PubMed DOI: 10.1056/ NEJM200102153440706

12. Dienstag J: The role of liver biopsy in chronic hepati- 
tis C. Hepatology. 2002,36: 152-160 PubMed https://doi. org/10.1053/jhep.2002.36381

13. Solis Herruzo JA. Current indications of liver biopsy. Rev. Esp Enferm Dig. 2006;98:122-139 PubMed .

14. Riley TR. How often does ultrasound marking change the liver biopsy site? Am J Gastroenterol. 1999; 94:3320 -3322 PubMed

15. Sparchez Z. Complications After Percutaneous Liver Biopsy in Diffuse Hepatopathies. Rom J Gastroenterol. 2005;14: 379 -383 PubMed.

16. Ferraioli G, Tinelli C, Dal Bello B, et al. Accuracy of real-time shear wave elastography for assessing liver fibrosis in chronic hepatitis C: a pilot study. Hepatology. 2012;56:2125-33 PubMed . https://doi.org/10.1002/ hep. 25936

17. Myers RP, Elkashab M, Ma M, Crotty P, Pomier-Layrargues G. 2010. Transient elastography for the noninvasive assessment of liver fibrosis: a multicentre Canadian study. Canadian Journal of Gastroenterology, 2411,
661. http://dx.doi.org/10.1155/2010/15398618- Ioan, S., Radu B, Roxana S, Monica L, Alina P, Mirela D, Alexandra D. 2011. How efficient is acoustic radiation force impulse elastography for the evaluation of liver stiffness? Hepatitis monthly, 20117, Jul, 532-538.

19. Leschied, J. R., Dillman, J. R., Bilhartz, J., Heider, A., Smith, E. A., \& Lopez, M. J. 2015. Shear wave elastography helps differentiate biliary atresia from other neonatal/infantile liver diseases. Pediatric radiology, 453, 366-375. DOI 10.1007/s00247-014-3149-z

20. Corpechot, C., El Naggar, A., Poujol-Robert, A., Ziol, M., Wendum, D., Chazouillères, O., \& Poupon, R. 2006. Assessment of biliary fibrosis by transient elastography in patients with PBC and PSC. Hepatology, 435, 1118-1124.https://doi.org/10.1002/hep.21151 21- Hanquinet S, Rougemont AL, Courvoisier D, et al. Acoustic radiation force impulse ARFI elastography for the noninvasive diagnosis of liver fibrosis in children. Pediatr Radiol 2013;43:545-51. PubMed DOI 10.1007/s00247012-2595-8 